Подписана в печать 30.09.2020

\title{
УРАВНЕНИЯ В НЕЧЕТКОЙ РАСПРЕДЕЛЕННОЙ LР-СТРУКТУРЕ И ВОЗМОЖНОСТИ ИХ ПРИМЕНЕНИЯ В ИНТЕЛЛЕКТУАЛЬНЫХ СИСТЕМАХ
}

\author{
() 2020 С. Д. Махортов ${ }^{\bowtie}$ \\ Воронежский государственный университет \\ Университетская пл., 1, 394018 Воронеж, Российская Федерация
}

\begin{abstract}
Аннотация. Алгебраическая теория LP-структур предлагает и обосновывает формальную методологию управления знаниями в интеллектуальных системах продукционного типа. Она обеспечивает эффективное решение ряда важных задач, связанных с продукционными системами. К таковым относятся эквивалентные преобразования, верификация, оптимизация баз знаний. Важный раздел теории составляет также метод релевантного обратного логического вывода (LP-вывод), направленный на снижение числа обращений к внешним источникам информации. Теоретической базой метода служит аппарат продукционно-логических уравнений в LP-структурах. Как показывает статистика экспериментов, решая уравнение и обрабатывая множество решений, можно добиться снижения числа внешних запросов в процессе вывода в среднем на 15-20 \%.

В статье определяется расширенный класс таких уравнений для алгебраической системы (FDLP-структуры), моделирующей распределенные системы знаний продукционного типа с нечеткими правилами. Анонсированы полученные автором результаты о разрешимости уравнений и методе их решения. Нахождение решения уравнения в FDLP-структуре соответствует обратному нечеткому логическому выводу в распределенной интеллектуальной системе. Рассмотренный класс уравнений создает основу для продвижений в области оптимизации нечеткого распределенного логического вывода и верификации соответствующих баз знаний. Предложены варианты стратегий обратного вывода с использованием нескольких параметров релевантности.

Еще одно из возможных приложений аппарата продукционно-логических уравнений - верификация баз знаний интеллектуальных систем. В работе сформулированы методика и общий алгоритм применения уравнений для верификации нечетких распределенных баз знаний в плане выявления противоречий и избыточности.

Следующими шагами исследований на рассматриваемом направлении являются уточнение и программная реализация применения новых параметров релевантности FDLP-вывода, статистический анализ результатов.

Ключевые слова: нечеткая продукционная система, распределенная система, алгебраическая модель, FDLP-структура, продукционно-логическое уравнение, релевантный обратный вывод, параметры релевантности, верификация знаний.
\end{abstract}

\section{ВВЕДЕНИЕ}

Алгебраические методы представляют эффективное средство формального построения и исследования моделей информационных систем широкого спектра, в том числе интеллектуальных [1-2]. Это положение в

Махортов Сергей Дмитриевич e-mail: msd_exp@outlook.com полной мере относится и к широко распространенным на практике логическим системам продукционного типа, которые представляют важное направление дисциплины искусственного интеллекта [3].

Обладая сравнительно несложной структурой, продукционные системы могут использоваться и в качестве «испытательного полигона» для создания и исследования методов управления знаниями, которые в Контент доступен под лицензией Creative Commons Attribution 4.0 License. The content is available under Creative Commons Attribution 4.0 License. 


\section{С. Д. Махортов}

дальнейшем могут быть применены для построения более сложных интеллектуальных систем, в том числе параллельных и распределенных [4-5].

В последнее десятилетие автором разрабатывается алгебраическая теория LP-структур (lattice production structures) [6-7], обеспечивающая эффективное решение задач, связанных с продукционными системами. К таковым относятся эквивалентные преобразования, верификация, оптимизация баз знаний, а также ускорение логического вывода.

В то же время важное свойство современных интеллектуальных систем представляет нечеткий характер знаний и рассуждений [8]. Поэтому возникла актуальная задача распространения преимуществ теории LP-структур на нечеткие продукционные системы. Начало этому направлению положено в статье [9]. В то же время прогресс информационных технологий приводит к значительному увеличению масштабов и сложности решаемых задач. Компьютерные системы становятся большими и распределенными [10], что также требует качественных продвижений в методах их построения.

В статьях [11-12] были сделаны соответствующие шаги в развитии LP-структур определена алгебраическая модель, выразительные возможности которой охватывают нечеткие распределенные продукционно-логические системы. Для понятия нечеткой распределенной LP-структуры (FDLP-структуры) рассмотрен стандартный круг вопросов: о замыкании, эквивалентных преобразованиях, канонической форме, логической редукции. Настоящая работа дополняет эту модель рассмотрением аппарата продукционно-логических уравнений. В результате создается теоретическая основа для оптимизации нечеткого распределенного логического вывода.

Статья состоит из следующих основных разделов. В п. 1 вводятся необходимые базовые понятия и обозначения, в качестве отправной точки формулируются имеющиеся математические результаты. В п. 2 вводится аппарат продукционно-логических уравнений в FDLP-структуре, анонсируются результаты об их разрешимости и методе решения.
В п. 3 излагаются основные идеи практического применения продукционно-логических уравнений для ускорения нечеткого распределенного обратного вывода (релевантного FDLP-вывода) и верификации соответствующих баз знаний. В Заключении подводятся итоги исследования и указываются некоторые перспективы.

\section{1. ОСНОВНЫЕ ПОНЯТИЯ ТЕОРИИ FDLP-СТРУКТУР}

Исходные для настоящего раздела положения теории решеток, нечетких множеств и бинарных отношений изложены, например, в [13-14]. Напомним некоторые из них, чтобы зафиксировать используемые обозначения.

Нечеткое множество $A=\left(F, \mu_{F}\right)$ определяется функцией принадлежности $\mu_{F}: F \rightarrow[0,1]$ на некотором (обычном) множестве $F$ (значение $\mu_{F}(a)$ называется степенью принадлежности $a$ к $S$ ). Нечеткое бинарное отношение $R$ на множестве $F$ - это нечеткое множество упорядоченных пар элементов из $F$ c заданной функцией принадлежности $\mu_{R}: F \times F \rightarrow[0,1]$.

Нечеткое бинарное отношение $R$ на произвольном множестве $F$ называется рефлексивным, если для любого $a \in F$ справедливо $\mu_{R}(a, a)=1$.

В целях моделирования нечеткого логического вывода будем использовать понятие композиции отношений в классической семантике - (max-min)-композицию. Для отношения $R$ на множестве $F$ отношение $R^{2}=R \circ R$ определяется следующим образом:

$$
\mu_{R^{2}}(a, c)=\max _{b}\left(\min \left(\mu_{R}(a, b), \mu_{R}(b, c)\right)\right),
$$

где $a, b, c \in F$.

Нечеткое бинарное отношение $R$ на множестве $F$ называется транзитивным, если для любых $a, b, c \in F \quad$ справедливо $\mu_{R}(a, c) \geq \min \left(\mu_{R}(a, b), \mu_{R}(b, c)\right)$. Существует замыкание произвольного нечеткого отношения относительно свойств рефлексивности и транзитивности. Обзор алгоритмов его построения представлен в [15].

Пусть дана атомно-порожденная решетка $\mathbb{F}$ [13], представляющая множество всех ко- 
Уравнения в нечеткой распределенной LP-структуре ...

нечных подмножеств некоторого универсума $F$. На $\mathbb{F}$ рассматривается (вторичное) нечеткое бинарное отношение $R$, содержащее $\supseteq$, а также обладающее транзитивностью и дистрибутивностью. Последнее свойство уточняется следующим определением.

Определение 1.1. Нечеткое бинарное отношение $R=\left(\mathbb{F}, \mu_{R}\right)$ на решетке $\mathbb{F}$ называется дистрибутивным, если для любых $A, B_{1}, B_{2} \in \mathbb{F}$ справедливо

$$
\mu_{R}\left(A, B_{1} \cup B_{2}\right) \geq \min \left(\mu_{R}\left(A, B_{1}\right), \mu_{R}\left(A, B_{2}\right)\right) .
$$

Отношение с упомянутыми выше тремя свойствами будем называть продукционно-логическим (для краткости - просто логическим). Эти свойства естественным образом отражают возможности нечеткого логического вывода.

Предлагаемый подход к исследованию интеллектуальных систем продукционного типа основан на представлении базы знаний (множества правил) в виде продукционно-логического отношения. Каждый элементарный факт отображается атомом решетки $\mathbb{F}$, предпосылка и заключение правила - соответствующими элементами решетки, а нечеткие правила представляются функцией принадлежности нечеткого бинарного отношения $R$.

Далее перейдем к определению алгебраической системы, моделирующей распределенную интеллектуальную систему. Каждому атому решетки $\mathbb{F}$ и каждой паре отношения $R$ (с положительным значением функции принадлежности) назначается множество узлов «вычислительной сети», на которых «хранится» этот атом и эта пара.

Пусть $N$ - множество узлов вычислительной сети, $\mathbb{N}$ - порожденный им булеан, то есть множество всех его подмножеств. Атомы решетки $\mathbb{F}$ и пары отношения $\mathrm{R}$ будут помечаться элементами решетки $\mathbb{N}$ как атрибутами.

На решетке $\mathbb{F}$ определено отображение Nodes(), которое каждому атому $a \in \mathbb{F}$ ставит в соответствие единственный непустой элемент $X \in \mathbb{N}(\operatorname{Nodes}(a)=X, X \neq \varnothing)$. На решетке $\mathbb{F}$ задается нечеткое бинарное отношение $R$. Кроме того, каждой паре $A, B \in \mathbb{F}$, для которой $\mu_{R}(A, B)>0$, также назначается непустой элемент $Y \in \mathbb{N}(\operatorname{Nodes}(A, B)=Y, Y \neq \varnothing)$.
Для моделируемой продукционной системы функция Nodes() определяет каждому элементарному факту или каждому правилу совокупность узлов распределенной вычислительной системы, где они хранятся.

Определение 1.2. Описанная выше алгебраическая система называется распределенной нечеткой LP-структурой (FDLP-структурой).

Замечание 1.1. При заданном отображении $\operatorname{Nodes}()$ решетка $\mathbb{F}$ и отношение $R$ также могут считаться распределенными.

Определение 1.3. Распределенное нечеткое бинарное отношение $R$ на распределенной решетке $\mathbb{F}$ называется продукционно-логическим, если оно рефлексивно, транзитивно и дистрибутивно, причем для каждой пары $A, B \in \mathbb{F}$ справедливо $\mu_{R}(A, B)>0 \Rightarrow$ $\Rightarrow \operatorname{Nodes}(A, B)=N$. Логическим замыканием отношения $R$ называется наименьшее продукционно-логическое отношение, содержащее $R$.

Последнее определение носит лишь теоретический характер и используется для обоснования других результатов, в том числе и с практической точки зрения.

Для понятия FDLP-структуры в работе [12] рассмотрен стандартный для теории LP-структур круг вопросов, а именно - о замыкании, эквивалентных преобразованиях, канонической форме, логической редукции. Приведем здесь результаты, необходимые для дальнейшего изложения.

В частности, справедливо следующее утверждение.

Теорема 1.1. Для каждого распределенного нечеткого бинарного отночения $R$ на распределенной решетке существует логическое замыкание $\bar{R}$.

Ha FDLP-структуру стандартным образом переносится концепция эквивалентных преобразований.

Определение 1.4. Два распределенных нечетких отношения $R, P$ на решетке $\mathbb{F}$ называются эквивалентными $(R \sim P)$, если их логические замыкания совпадают. Эквивалентным преобразованием отношения $R$ называется такая модификация функции принад- 


\section{С. Д. Махортов}

лежности $\left(\mu_{R} \rightarrow \mu_{P}\right)$, что новое отношение $P$ эквивалентно $R$.

Наряду с функцией Nodes() введем еще два отображения из $\mathbb{F}$ в $\mathbb{N}$. Пусть $A \in \mathbb{F}$. Тогда, поскольку решетка $\mathbb{F}$ атомно-порожденная, существует представление $A=\bigcup a_{i}$ в виде объединения атомов [13]. Обозначим

$$
\begin{aligned}
& \operatorname{NodesMeet}(A)=\bigcap_{i} \operatorname{Nodes}\left(a_{i}\right) ; \\
& \operatorname{Nodes} \operatorname{Join}(A)=\bigcup_{i} \operatorname{Nodes}\left(a_{i}\right) .
\end{aligned}
$$

В терминологии моделируемой предметной области отображение NodesMeet() определяет вычислительные узлы, каждый из которых хранит все элементарные факты, составляющие множество фактов $A$. Отображение NodesJoin() выдает все узлы, хранящие хотя бы один из таких фактов.

Пусть на некотором вычислительном узле работает локальная машина вывода [16], оперирующая в основном фактами этого узла. Для нее окажется затратным поиск на других узлах сети правил для вывода собственных фактов. Заранее неизвестно, какие узлы предстоит запросить и в каком порядке. Поэтому естественным окажется хранение на узле всех правил, оперирующих фактами этого же узла. Таким образом, приходим к следующим понятиям целесообразного распределения правил по узлам вычислительной сети.

Определение 1.5. Распределенное нечеткое отношение $R$ на решетке $\mathbb{F}$ называется релевантно-корректным, если для любых $A, B \in \mathbb{F}$, $\mu_{R}(A, B)>0, \quad$ справедливо $\operatorname{Nodes}(A, B) \supseteq$ $\supseteq \operatorname{NodesJoin}(A) \cup$ NodesJoin $(B) ;$ релевантнонормализованньм, если для нее $\operatorname{Nodes}(A, B)=$ $=\operatorname{NodesJoin}(A) \bigcup N o d e s J o i n(B)$.

Оказывается, что произвольное отношение может быть приведено к некоторому упрощенному виду.

Определение 1.6. Распределенное нечеткое отношение $R$ на распределенной решетке $\mathbb{F}$ называется каноническим, если оно релевантно-нормализовано, а его функция принадлежности положительна лишь на парах вида $(A, a)$, где $A \in \mathbb{F}, a-$ атом в $\mathbb{F}$.

В работе [12] доказан следующий факт.

Теорема 1.2. Для любого распределенного нечеткого отночения существует эквивалентное ему каноническое отночение.

\section{2. ПРОДУКЦИОННО-ЛОГИЧЕСКИЕ УРАВНЕНИЯ В FDLР-СТРУКТУРЕ}

В этом разделе вводится связанный с FDLP-структурами класс уравнений. Нахождение решения уравнения соответствует обратному нечеткому логическому выводу в распределенной базе знаний.

Пусть дано нечеткое распределенное отношение $R$ на распределенной решетке $\mathbb{F}$ и имеет место $\mu_{R}(A, B)>0$ для некоторых элементов $A, B \in \mathbb{F}$. Тогда $B$ может быть назван образом $A$, а $A-$ прообразом $B$ при отношении $R$. Каждый элемент решетки может иметь много образов и прообразов с различной степенью принадлежности (значением $\left.\mu_{R}(A, B)\right)$.

Для данного $B \in \mathbb{F}$ минимальным прообразом при отношении $R$ называется такой $A \in \mathbb{F}$, что $\mu_{R}(A, B)>0$ и $A$ является минимальным в том смысле, что не содержит никакого другого $A_{1} \in \mathbb{F}$, для которого $\mu_{R}\left(A_{1}, B\right)>0$.

Определение 2.1. Атом $x \in \mathbb{F}$ называется начальным (при отношении $R$ ), если нет ни одной пары $A, B \in \mathbb{F}$ такой, что $\mu_{R}(A, B)>0$, причем $x$ содержится в $B$ и не содержится в $A$. Элемент $X$ называется начальным, если все его атомы являются начальными. Подмножество $\mathbb{F}_{0}(R)$ (для краткости - просто $\mathbb{F}_{0}$ ), состоящее из всех начальных элементов $\mathbb{F}$, называется начальным множеством решетки $\mathbb{F}$ при отношении $R$.

Замечание 2.1. Начальные атомы решетки соответствуют в моделируемой продукционной системе таким элементарным фактам, которые не могут быть выведены с помощью правил (начальным фактам). Их истинность может быть определена только обращением к внешнему источнику информации (базе данных или пользователю).

Пусть $\bar{R}$ - логическое замыкание отношения $R$ (см. п. 1). Рассмотрим уравнение

$$
\bar{R}(X)=B
$$

где $B \in \mathbb{F}-$ заданный элемент, $X \in \mathbb{F}-$ неизвестный.

Определение 2.2. Приближенным (распределенным) решением уравнения (1) называется любой прообраз элемента $B$ в $\mathbb{F}_{0}$ при отношении $\bar{R}$. Решением (точным распределен- 
Уравнения в нечеткой распределенной LP-структуре ...

ным) (1) называется любой минимальный прообраз элемента $B$ в $\mathbb{F}_{0}$. Общим (распределенным) решением уравнения называется совокупность всех его решений $\left\{X_{s}\right\}, s \in S$. Распределенное решение $X$ называется локализованным, если $\operatorname{NodesMeet}(X) \neq \varnothing$.

Уравнения вида (1) будем называть продукиионно-логическими уравнениями в FDLP-структуре.

Фактором, усложняющим как обоснование метода решения (1), так и саму постановку этой задачи, является нечеткость отношения $R$. Помимо требуемой в определении 2.2 минимальности искомого прообраза $X$, необходимо учитывать и вторую его характеристику - значение функции принадлежности $\mu_{\bar{R}}(X, B)$.

Конечно, лучшими решениями будут дающие большее значение функции принадлежности, соответственно в моделируемой продукционной системе - результаты логического вывода с более высоким коэффициентом уверенности. Как вариант можно попробовать в некоторой мере пожертвовать точностью решения в пользу повышения коэффициента уверенности до практически приемлемых результатов. Детальному теоретическому исследованию данного вопроса в будущем, возможно, помогут методы многокритериальной оптимизации.

Для начала будем рассматривать простейшую постановку задачи для (1). Будем искать такие решения, на которых функция принадлежности принимает положительные значения. Однако для каждого решения $X$ эта величина $\left(\mu_{\bar{R}}(X, B)\right)$ должна быть вычислена.

В проведенных автором исследованиях получены положительные результаты, касающиеся вопросов разрешимости уравнений вида (1) в нечеткой LP-структуре, а также обоснованы способы их решения. Эти исследования составят предмет отдельных публикаций. Принципиально новым в настоящей работе является распределенный характер отношения $R$. Однако данное обстоятельство не оказывает какого-либо существенного влияния на получение упомянутых теоретических результатов.
Аппарат уравнений в FDLP-структуре создает основу для продвижений в области оптимизации нечеткого распределенного логического вывода и верификации соответствующих баз знаний.

\section{3. О ПРАКТИЧЕСКОМ ПРИМЕНЕНИИ FDLР-УРАВНЕНИЙ}

Изложим идеи практического применения аппарата продукционно-логических уравнений для оптимизации нечеткого распределенного обратного вывода (релевантного FDLP-вывода) в интеллектуальных системах.

Для таких систем, особенно распределенных, существенную роль играет организация хранения фактов. В то время как множество правил нередко может целиком располагаться в основной памяти компьютера, для начальных фактов (см. замечание 2.1) это возможно далеко не всегда. Например, база знаний «Здоровье» [16] может применяться для тестирования пациентов, количество которых не ограничено, и каждый из них имеет собственную исходную информацию. Единственная операция чтения с диска или, более того, получения информации по сети или у интерактивного пользователя, по времени выполнения способна перекрыть тысячи операций обращения к основной памяти. Таким образом, возникает потребность в такой организации процесса логического вывода, который потребует как можно меньше внешних запросов.

При обычном обратном выводе [16] после выбора исходной гипотезы машина вывода осуществляет просмотр содержащих гипотезу заключений правил, переходя к предпосылкам и в свою очередь рекурсивно проверяя их истинность. Когда в данном процессе в качестве очередной гипотезы встречается начальный факт (он не появляется в заключениях правил), для проверки его истинности происходит обращение к диску или пользователю. При этом в конечном итоге не все получаемые начальные факты оказываются необходимыми для осуществления логического вывода. 


\section{С. Д. Махортов}

Стратегия релевантного вывода направлена на минимизацию количества медленно выполняемых запросов [7]. Запросы по возможности должны соответствовать лишь тем фактам, которые действительно необходимы при выводе. Отрицательный ответ на единичный запрос исключает последующие запросы об элементах связанного подмножества фактов - прообраза гипотезы. Кроме того, при LP-выводе предпочтение отдается тестированию множеств фактов минимальной мощности.

Первая стадия обратного вывода на основе продукционно-логического уравнения (1) состоит в его решении - построении всех минимальных начальных прообразов в FDLP-структуре для атомов, соответствующих значениям объекта экспертизы - гипотезе. Далее в построенном множестве достаточно найти тот прообраз, который отображает лишь истинные факты, после чего сразу можно сделать заключение о соответствующем значении объекта экспертизы.

Простой путь в этом направлении - просматривать прообразы последовательно, задавая пользователю вопросы о соответствующих начальных фактах (или обращаясь к базе данных за этими фактами). Этот способ дает некоторые преимущества - исследуются лишь минимальные прообразы. Также предварительно можно исключить из процесса противоречивые прообразы, то есть содержащие несовместимые начальные факты (например, альтернативные значения одного и того же объекта [16]).

Однако существует более эффективный способ - приоритетный просмотр прообразов, содержащих значения наиболее «релевантных» объектов. Таковыми в первую очередь считаются объекты, чьи значения присутствуют в максимальном количестве построенных прообразов. Тогда единственный отрицательный ответ на заданный вопрос исключает из рассмотрения сразу большое количество прообразов, что соответственно ускоряет исследование. Второй показатель релевантности тестируемого объекта - присутствие его значений в прообразах минимальной мощности. Таким образом, предпо- чтение отдается тем прообразам, проверка истинности которых потребует меньшего количества вопросов пользователю (или обращений к базе данных).

Сочетая указанные два показателя релевантности, можно достичь результатов, по эффективности существенно превышающих возможности стандартной машины вывода. Эксперименты показывают [6], что при применении релевантного LP-вывода снижение числа выполняемых медленных запросов достигает в среднем 15-20\%.

Нечеткий и распределенный характер базы знаний интеллектуальной системы создает дополнительные возможности (и трудности) повышения эффективности логического вывода.

К задачам моделирования нечеткой продукционной системы относится достижение за приемлемое время для доказываемой гипотезы более высокого значения коэффициента уверенности. Таким образом, при реализации стратегий нечеткого релевантного LP-вывода, дополнительно к отмеченным выше двумя характеристиками, необходимо учитывать еще один параметр релевантности начальных атомов решетки. Он представляет значение функции принадлежности, вычисляемое для каждого найденного решения уравнения вида (1).

Еще одна очевидная цель моделирования интеллектуальных систем рассматриваемого класса - снижение трафика между узлами вычислительной сети в процессе выполнения логического вывода. Стратегия релевантного FDLP-вывода, наряду с уже упомянутыми тремя параметрами релевантности, должна использовать дополнительные показатели. Они связаны с атрибутами хранения фактов и правил, которые описываются рассмотренными в п. 1 настоящей работы отображениями Nodes(), NodesMeet(), NodesJoin(). Эти показатели, в частности, могут характеризовать максимальный «разброс» и/или максимальную локализацию атомов исследуемого прообраза в узлах сети.

Простейший вариант алгоритма определения наиболее релевантных начальных атомов решетки в процессе FDLP-вывода состо- 
Уравнения в нечеткой распределенной LP-структуре ...

ит в элементарном суммировании значений используемых показателей. При этом имеется широкое поле для практических экспериментов, комбинирующих четыре или более параметра релевантности с различными «весами», выбираемыми в зависимости от задач, стоящих в конкретной предметной области. Возможны также более глубокие теоретические обоснования стратегий подсчета релевантности атомов, например, на основе методов многокритериальной оптимизации.

На основе теории FDLP-структур и решения продукционно-логических уравнений могут быть также организованы методы верификации баз знаний. Во-первых, с помощью логической редукции FDLP-структуры база знаний может быть эквивалентно минимизирована [12]. Таким образом, в ней могут быть выявлены и устранены избыточные правила.

Во-вторых, базу знаний можно формально исследовать на противоречивость с помощью следующего алгоритма. Сформулируем противоречивую гипотезу $B$, содержащую несовместимые факты (например, составленную из пары альтернативных значений объекта экспертизы [16]). Далее решим продукционно-логическое уравнение (1) с данной гипотезой в качестве правой части. Если в результате получится хотя бы одно непустое непротиворечивое решение $X$ с достаточно высоким значением функции принадлежности $\mu_{\bar{R}}(X, B)$, можно сделать вывод о некорректности (противоречивости) базы знаний.

\section{ЗАКЛЮЧЕНИЕ}

В настоящей работе определен класс продукционно-логических уравнений в обобщенной LP-структуре (FDLP-структуре), pacширяющей область применения этой алгебраической теории до нечетких распределенных интеллектуальных систем продукционного типа.

Анонсированы результаты о разрешимости и способе решений этих уравнений. Нахождение решения продукционно-логического уравнения соответствует обратному нечеткому логическому выводу на распределенной решетке. Аппарат уравнений в
FDLP-структуре создает основу для продвижений в области оптимизации нечеткого распределенного логического вывода и верификации соответствующих баз знаний.

Предложена концепция нечеткого распределенного релевантного LP-вывода (FDLP-вывода). Описаны стратегии выбора параметров релевантности.

Сформулированы методика и алгоритм применения аппарата продукционно-логических уравнений для верификации нечетких распределенных баз знаний.

Следующими шагами исследований на рассматриваемом направлении являются уточнение и программная реализация применения новых показателей релевантности FDLP-вывода, статистический анализ результатов.

Исследование выполнено при финансовой поддержке РФФИ в рамках научного проекта № 19-07-00037.

\section{КОНФЛИКТ ИНТЕРЕСОВ}

Автор декларирует отсутствие явных и потенциальных конфликтов интересов, связанных с публикацией настоящей статьи.

\section{СПИСОК ЛИТЕРАТУРЫ}

1. Oles, F. J. An Application of Lattice Theory to Knowledge Representation / F. J. Oles // Theor. Comput. Sci. - 2000, 249, 1. - P. 163-196.

2. Бениаминов, Е. М. Алгебраические методы в теории баз данных и представлении знаний / Е. М. Бениаминов. - М. : Научный мир, 2003. - 184 c.

3. Zhozhikashvili, A. V. Categories for Description of Dynamic Production Systems / A. V. Zhozhikashvili, V. L. Stefanuk // Proceedings of Joint Conference on Knowledge-Based Software Engineering JCKBSE-2006. Edited by Enn Tyugu, Takahira Yamaguchi. - Amsterdam : IOS Press, 2006. - P. 285-293.

4. Ishida, T. Parallel, Distributed and Multiagent Production Systems / T. Ishida. - Lecture Notes in Computer Science, 1994. - 166 p.

5. Сошников, Д. В. Логический вывод на основе удалённого вызова и включения в си- 


\section{С. Д. Махортов}

стемах с распределенной фреймовой иерархией / Д. В. Сошников. - М. : Вузовская книга, 2002. - 48 с.

6. Махортов, С. Д. Математические основы искусственного интеллекта: теория LP-структур для построения и исследования моделей знаний продукционного типа / С. Д. Махортов ; Под ред. В. А. Васенина. - М. : МЦНМО, 2009. - 304 c.

7. Махортов, С. Д. Релевантный обратный вывод и верификация логических программ на основе решения уравнений в LP-структурах / С. Д. Махортов // Методы и средства обработки информации: III Всероссийская научная конференция. Москва, 6-8 октября 2009 г.: Труды конф. / Под ред. Л. Н. Королева. - М. : ВМиК МГУ, 2009. - С. 143-148.

8. Батыршин, И. 3. Нечеткие гибридные системы: Теория и практика / И. 3. Батыршин, А. О. Недосекин, А. А. Стецко, В. Б. Тарасов, А. В. Язенин, Н. Г. Ярушкина ; Под ред. Н. Г. Ярушкиной. - М. : Физматлит, 2007. $208 \mathrm{c}$.

9. Махортов, С. Д. Оптимизация метода LP-вывода / С. Д. Махортов, А. Н. Шмарин // Нейрокомпьютеры. Разработка, применение. - 2013, № 9. - С. 59-63.

10. Васенин, B. А. Средства суперкомпьютерных систем для работы с агент-ориентиро- ванными моделями / В. А. Васенин, В. Л. Макаров, А. Р. Бахтизин, В. А. Роганов, И. А. Трифонов // Программная инженерия. - 2011, № 3. - C. 2-14.

11. Махортов, С. Д. Алгебраическая модель интеллектуальной системы с нечеткими правилами / С. Д. Махортов // Программная инженерия. - 2019. - № 11-12. - С. 457-463.

12. Махортов, С. Д. Алгебраическая модель распределенной продукционной системы с нечеткими правилами / С. Д. Махортов, М. В. Лещинская // // Вестник Воронеж. гос. ун-та. Сер. Системный анализ и информационные технологии. - 2019. - № 3. - С. 149-157.

13. Birkhoff, G. Lattice Theory / G. Birkhoff. Third Edition. - Providence: Am. Math. Soc., 1967. - $423 \mathrm{p}$.

14. Рыжов, А. П. Элементы теории нечетких множеств и ее приложений / А. П. Рыжов. - М. : Диалог-МГУ, 2003. - 81 с.

15. Garmendia, L. An Algorithm to Compute the Transitive Closure, a Transitive Approximation and a Transitive Opening of a Fuzzy Proximity / L. Garmendia, R.G. Del Campo, V. López, J. Recasens // Mathware \& Soft Computing. 2009, No. 16. - P. 175-191.

16. Sowyer, B. Programming Expert Systems in Pascal / B. Sowyer, D. Foster. - John Wiley \& Sons, Inc., 1986. - $186 \mathrm{p}$.

Махортов Сергей Дмитриевич - д-р физ.-мат. наук, доц., зав. кафедрой программирования и информационных технологий Воронежского государственного университета. E-mail: msd_exp@outlook.com ORCID iD: https://orcid.org/0000-0001-6362-4468 


\title{
EQUATIONS IN A FUZZY DISTRIBUTED LP-STRUCTURE AND THE POSSIBILITY OF THEIR APPLICATION IN INTELLIGENT SYSTEMS
}

\author{
(c) 2020 S. D. Makhortov ${ }^{\bowtie}$ \\ Voronezh State University \\ 1, Universitetskaya Square, 394018 Voronezh, Russian Federation
}

\begin{abstract}
Annotation. The algebraic theory of LP-structures proposes and substantiates a formal methodology for knowledge management in intelligent production-type systems. It provides an effective solution to a number of important problems associated with production systems. These include equivalent transformations, verification, optimization of knowledge bases. An important section of the theory is also the method of relevant backward logical inference (LP-inference) aimed at reducing the number of calls to external sources of information. The theoretical basis of the method is the apparatus of production-logical equations in LP-structures. As the statistics of experiments show, when solving the equation and processing a set of solutions, it is possible to reduce the number of external requests during the inference process by an average of 15-20\%.

The article defines and investigates an extended class of such equations for an algebraic system (FDLP-structure) that models distributed knowledge systems of production type with fuzzy rules. The results obtained by the author on the solvability of the equations and the method for their solution are announced. Finding a solution to an equation in an FDLP-structure corresponds to backward fuzzy inference in a distributed intelligent system. The considered class of equations creates the basis for advances in the field of optimization of fuzzy distributed inference and in verification of the corresponding knowledge bases. Variants of backward inference strategies using several relevance parameters are proposed.

Another possible application of the apparatus of production-logical equations is the verification of knowledge bases of intelligent systems. The paper formulates a methodology and a general algorithm for applying equations to the verification of fuzzy distributed knowledge bases in terms of identifying contradictions and redundancy.

The next steps of research in the direction under consideration are the refinement and software implementation of the application of the new relevance parameters of the FDLP-inference, and the statistical analysis of the results.
\end{abstract}

Keywords: fuzzy production system, distributed system, algebraic model, FDLP-structure, production-logical equation, relevant backward inference, relevance parameters, knowledge verification.

\section{CONFLICT OF INTEREST}

The author declare the absence of obvious and potential conflicts of interest related to the publication of this article.

\section{REFERENCES}

1. Oles F. J. An Application of Lattice Theory to Knowledge Representation. Theor. Comput. Sci. 2000. 249 (1). 163-196.

Makhortov Sergey D.

e-mail: msd_exp@outlook.com
2. Beniaminov E. M. Algebraic methods in the theory of databases and knowledge representation. Moscow, Nauchnyj mir. 2003 (in Russian)

3. Zhozhikashvili A. V. \& Stefanuk V. L. Categories for Description of Dynamic Production Systems, In: Tyugu, E. \& Yamaguchi, T. (ed.) Proceedings of Joint Conference on Knowledge-Based Software Engineering JCKBSE-2006, Amsterdam, IOS Press, 2006. pp. 285-293.

4. Ishida T. Parallel, Distributed and Multiagent Production Systems. Lecture Notes in Computer Science. 1994.

5. Soshnikov D. V. Logical Inference Based on Remote Call and Inclusion in Distributed Frame 


\section{С. Д. Махортов}

Hierarchy Systems. Moskow, High School Book. 2002. (in Russian)

6. Makhortov S. D. Mathematical Foundations of Artificial Intelligence: The LP structures theory for the knowledge models of production type construction and research. Edts. by V. A. Vasenin. Moscow, MCCME. 2009. (in Russian)

7. Makhortov S. D. The Relevant Backward Inference and Verification of Logic Programs Based on the Solution of Equations in LP Structures, In: Korolev, L. N. (ed.) Proceedings of Third All-Russian scientific conference "Methods and means of information processing", Moskow, MSU, 2009. pp. 143-148. (in Russian)

8. Batyrshin I. Z. et al. The Fuzzy Gibrid Systems: Theory and Practice. Moskow, Fizmatlit. 2007. (in Russian)

9. Makhortov S. D. \& Shmarin A. N. Optimizing of LP inference method. Nejrokomp'jutery. Razrabotka, primenenie. 2013. 9. P. 59-63 (in Russian).

10. Vasenin V. A. et al. Supercomputer System Tools for Working with Agent-Based Models. Programmnaya inzheneriya. 2011.3. P. 2-14. (in Russian)
11. Makhortov S. D. An Algebraic Model of the Intellektual System with Fuzzy Rules. Programmnaya inzheneriya. 2019. 12. P. 32-38. (in Russian)

12. Mahortov S. D. \& Leshchinskaya M. V. An Algebraic Model of the Distributed Intellektual System with Fuzzy Rules. Vestnik VGU. Serija Sistemnyj analiz i informacionnye tehnologii. 2019. 3. P. 149-157. (in Russian)

13. Birkhoff G. Lattice Theory. Third Edition. Providence, Am. Math. Soc. 1967.

14. Ryzhov A. P. Elements of the Theory of Fuzzy Sets and of its Applications. Moskow, Dialog-MGU. 2003. (in Russian).

15. Garmendia L., Del Campo R. G., López $V$. \& Recasens J. An Algorithm to Compute the Transitive Closure, a Transitive Approximation and a Transitive Opening of a Fuzzy Proximity. Mathware \& Soft Computing. 2009. 16. P. 175191.

16. Sowyer B. \& Foster D. Programming Expert Systems in Pascal. John Wiley \& Sons, Inc. 1986.

Makhortov Sergey D. - DSc in Computer Sciences, Head of Programming and Information Technology Department at Voronezh State University, Russia.

E-mail:msd_exp@outlook.com

ORCID iD: https://orcid.org/0000-0001-6362-4468 